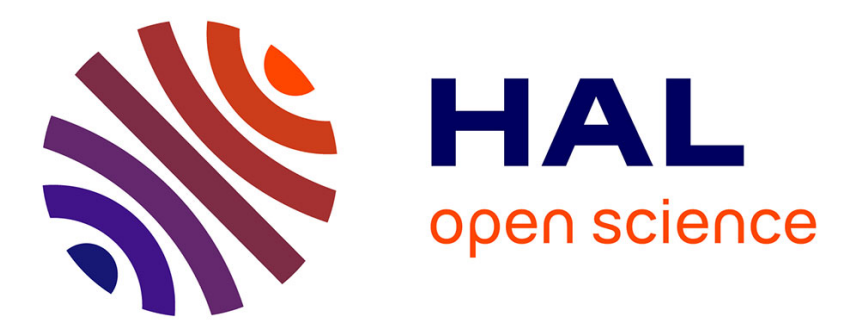

\title{
A two-dimensional liquid-like phase on Ga-rich GaN (0001) surfaces evidenced by first principles molecular dynamics
}

Kieu My Bui, Mauro Boero, Kenji Shiraishi, Atsushi Oshiyama

\section{- To cite this version:}

Kieu My Bui, Mauro Boero, Kenji Shiraishi, Atsushi Oshiyama. A two-dimensional liquid-like phase on Ga-rich GaN (0001) surfaces evidenced by first principles molecular dynamics. Japanese Journal of Applied Physics, 2020, 59 (SG), pp.SGGK04. 10.7567/1347-4065/ab650b . hal-02990661

\section{HAL Id: hal-02990661 \\ https://hal.science/hal-02990661}

Submitted on 22 Dec 2020

HAL is a multi-disciplinary open access archive for the deposit and dissemination of scientific research documents, whether they are published or not. The documents may come from teaching and research institutions in France or abroad, or from public or private research centers.
L'archive ouverte pluridisciplinaire HAL, est destinée au dépôt et à la diffusion de documents scientifiques de niveau recherche, publiés ou non, émanant des établissements d'enseignement et de recherche français ou étrangers, des laboratoires publics ou privés. 


\title{
A two-dimensional liquid-like phase on Ga-rich GaN (0001) surfaces evidenced by first principles molecular dynamics
}

\author{
Kieu My Bui ${ }^{1}$, Mauro Boero ${ }^{1,2}$, Kenji Shiraishi ${ }^{1}$ and Atsushi Oshiyama ${ }^{1}$ \\ ${ }^{1}$ Institute of Materials and Systems for Sustainability, Nagoya University, Furo-cho, Chikusa, \\ Nagoya 464-8601 Japan \\ ${ }^{2}$ University of Strasbourg, Institut de Physique et Chimie des Matériaux de Strasbourg \\ (IPCMS), CNRS, UMR 7504, 23 rue du Loess, F-67034 Strasbourg, France \\ E-mail: oshiyama@imass.nagoya-u.ac.jp, mauro.boero@ipcms.unistra.fr
}

First principles molecular dynamics simulations have been used as a tool to investigate at an atomistic level the finite temperature behavior of a typical GaN growing surface. At $1300 \mathrm{~K}$, Ga adatoms show a high degree of destabilization and a departure of the diffusivity from an ordinary Arrhenius trend. Two signatures, a drastic change in the radial distribution function and a sudden increase of the diffusion constant, allow to quantify such a diffusion process. Such a high diffusivity results in the arising of a pronounced peak at about $2.6 \AA$, typical of Ga-Ga bonds, indicating a tendency to cluster together of the Ga adatoms. Along with the remarkable diffusivity observed at $1300 \mathrm{~K}$, this result indicates rather clearly that at the typical experimental temperature, the epitaxial growth occurs not on a rigid surface in which Ga adatoms are tightly bound to specific sites, but rather on a two-dimensional liquid state.

\section{Introduction}

Gallium nitride $(\mathrm{GaN})$, a fundamental material worldwide used for high performing optoelectronic devices, is rapidly becoming the primary candidate to unseat silicon ( $\mathrm{Si}$ ) in power electronics. In comparison to $\mathrm{Si}$, its wider band gap results in a superior ability to sustain higher voltages and, simultaneously, the higher mobility and robustness under harsh environments allow for faster current flow and outstanding stability, respectively [1-3]. As a consequence, GaN-based power devices are expected to be more efficient and less energy consuming than their Si-based counterparts $[1,4]$. Yet, their practical realization asks for the realization of thin films of higher quality with respect to what is done nowadays for 
optoelectronic applications. To this aim, the metalorganic vapor phase epitaxy (MOVPE) technique has been proven to be particularly suitable for the realization of high quality $\mathrm{GaN}$ films [4]. In MOVPE, the typical gas-phase precursors are trimethylgallium (TMG) and ammonia, delivered to the exposed area of the growing material by a carrier gas, either $\mathrm{H}_{2}$ or $\mathrm{N}_{2}$. On both the experimental and the theoretical fronts, intensive research activities have evidenced that the precursor molecule, trimethylgallium (TMG), commonly employed to produce these films, undergoes a decomposition in the gas phase [5]. Kangawa and his collaborators evidenced that a Ga rich surface is realized under $\mathrm{N}_{2}$ carrier gas condition [6,7]. On the theoretical front, recent first principle simulations in our group could provide a detailed atomic-level picture of a $\mathrm{Ga}$ rich surface, showing that Ga-Ga weak bond are realized on the exposed surface and these can become active sites for subsequent $\mathrm{N}$ incorporation $[8,9]$. Yet, a fundamental knowledge about both the actual microscopic structure of the Ga-rich growing surface and the $\mathrm{NH}_{3}$ decomposition and incorporation in the growing material at growth temperature is demanded. Since these details escape any experimental probe, we resort to first principles dynamical approaches, jointly with free energy sampling techniques, and the intensive use of high-performance computing (HPC) resources to shed some light into the properties of $\mathrm{Ga}$ atoms (adatoms) accumulating onto the GaN (0001) surface during the MOVPE growing process. These approaches are worldwide acknowledged as reliable virtual experiments able to extend and complement traditional laboratory investigations.

We evidence the arising of a high mobility of these atoms in the temperature range where actual experiments are performed $(1300 \mathrm{~K})$, which, in turn, is responsible for the formation of a Ga liquid-like state at the (0001) surface of the growing film.

\section{Computational methods}

Our simulations are done within the density functional theory (DFT) framework [10,11] and dynamical simulations are performed according to the method proposed in 1985 by Car and Parrinello [12-14] as implemented in the CPMD code [15]. Complementary calculations have been done with the real-space code (RSDFT) developed by our group [1618]. The temperature control was ensured by a velocity rescaling algorithm [19], with a 
fictitious electronic mass of 340 a.u. and an integration step of 4.0 a.u. ensuring good conservation of the constants of motion all along the dynamics. The Blue-Moon ensemble (BME) [17] was used to sample the free energy landscape and reaction path responsible for the migration and clustering of $\mathrm{Ga}$ adatoms at the surface of the system. The latter is composed of $96 \mathrm{GaN}$ formula units, constituting a slab of six $\mathrm{GaN}$ layers in which the bottom side is fixed to the bulk crystallographic positions and passivated with H-like atoms to mimic the thick substrate. On the opposite side, the surface carries four Ga adatoms, $\mathrm{Ga}(\mathrm{ad})$ as shown in the inset of Fig. 1. For the exchange and correlation part of the electronic interaction, we adopt the Perdew-Burke-Enrzerhof functional [21,22] complemented with the long-range van der Waals part proposed by Grimme [23]. Only valence electrons are explicitly treated, expanded on a plane-wave basis set with a cut-off of $70 \mathrm{Ry}$, while core electrons are replaced by Goedecker-Teter-Hutter [24] analytical norm-conserving pseudopotentials.

\section{Results and discussion}

\subsection{Results}

Starting from the computational model described in the previous paragraph and sketched in the inset of Fig.1, we first relaxed the structure via damped dynamics, to obtain a stable system. Then this system was gradually heated up from room temperature to $1300 \mathrm{~K}$, as shown in that same figure. This final target temperature corresponds to the experimental one at which the MOVPE growth of the material is realized. During this stage, we monitored the behavior of the system at each temperature plateau by computing the pair correlation functions (PCFs):

$$
g(r)=\frac{V}{4 \pi r^{2} N^{2}}\left\langle\sum_{i} \sum_{j \neq i} \delta\left(r-r_{i j}\right)\right\rangle
$$

being $N$ the number of atoms contained in a volume $V$ and $r_{i j}$ the interatomic distance. The average is computed on the trajectory produced by the dynamical simulation and, since both positions and velocities become simultaneously available, another quantity describing the dynamics, the diffusion coefficient can be directly obtained upon integration of the velocityvelocity autocorrelation function $[25,26]$ : 


$$
D=\frac{1}{3} \int_{0}^{t \rightarrow \infty}\left\langle\frac{1}{N} \sum_{I=1}^{N} v_{I}(t) v_{I}(0)\right\rangle d t
$$

The thermal fluctuations of the Ga-rich GaN structure are particularly interesting at the surface, specifically on the $\mathrm{Ga}(\mathrm{ad})$. In fact, here atoms oscillate more freely in comparison with $\mathrm{Ga}$ and $\mathrm{N}$ atoms constituting the bulk inner layers beneath the surface, and this enhancement of the thermal motion is particularly evident in the weakly bound $\mathrm{Ga}(\mathrm{ad})$ as detailed in the next paragraph. At room temperature, as shown in the bottom panel of Fig. 2, a sharp peak at $6.4 \AA$ in the pair correlation function (PCF) for $\mathrm{Ga}(\mathrm{ad})$ is the signature of a relative high stability of $\mathrm{Ga}$ adatoms on the sites where they have been placed in the construction of the model (inset of Fig. 1). However, at increasing temperature, this peak broadens significantly from 500 to $1100 \mathrm{~K}$. At $1300 \mathrm{~K}$, the large fluctuations that $\mathrm{Ga}(\mathrm{ad})$ adatoms undergo and the fact that, being less constrained than the bulk, they adsorb most of the kinetic and entropy contributions are the two main factors responsible for the disruption of the weak bond between the $\mathrm{Ga}(\mathrm{ad})$ and the subsurface $\mathrm{Ga}$ on the (0001) surface. Hence, Ga adatoms are dislodged and start migrating on the surface. This result is well represented by the $\mathrm{Ga}(\mathrm{ad})-\mathrm{Ga}(\mathrm{ad}) \mathrm{PCF}$ at $1300 \mathrm{~K}$ that extends from 2.0 to $6.5 \AA$ with a peak centered at 2.6-2.7 $\AA$. This peak is a clear evidence of the formation of chemical bonds between two $\mathrm{Ga}(\mathrm{ad})$ atoms and its corresponding spread accounts rather well for all the possible $\mathrm{Ga}(\mathrm{ad})$ Ga (ad) chemical bonds, from single $(\sim 2.59 \AA)$ to double $(\sim 2.41-2.25 \AA)$ and triple $(\sim 2.45$ $2.21 \AA$ ) bonds [27]. The long tail extending rather uniformly up to the borders of the simulation cell, visible both in the upper panel of Fig. 2 and in Fig. 3, resemble indeed what can be observed in non-polar liquids [28,29].

What can be inferred from the analysis of the PCFs is that $\mathrm{Ga}(\mathrm{ad})$ atoms, destabilized by the high temperature, start wandering on the GaN (0001) surface and due to their relatively high density, Ga adatoms can easily meet and start clustering, making structures that, as far as we could check within the size and time limits of the simulation, can extend up to trimers. Such an enhanced mobility is confirmed by the diffusion coefficient $D[21,22]$ computed at the various temperatures explored. The result of this analysis is summarized in Fig. 4: A feature that can be immediately remarked is that, at variance with lower temperatures, $D$ deviates from the linear behavior, on a logarithmic scale, typical of an Arrhenius behavior, when $T=1300 \mathrm{~K}$. This increased diffusivity corroborates the evidence about the enhanced mobility 
of $\mathrm{Ga}(\mathrm{ad})$ units, which behave like a liquid state, thus providing further support to the picture offered by the PCFs. Indeed, if we neglect the value of the diffusivity at $1300 \mathrm{~K}$, we can accommodate all the points of Fig. 4 in a linear fit (dashed red line in the figure) of the type

$$
\ln D=\ln D_{0}-E_{\mathrm{b}} / \mathrm{k}_{\mathrm{B}} T
$$

with the values of $E_{\mathrm{b}}=0.28 \mathrm{eV}$ and $D_{0}=7.94 \times 10^{-5} \mathrm{~cm}^{2} / \mathrm{s}\left(\mathrm{k}_{\mathrm{B}}\right.$ : the Boltzmann constant $)$.

Instead, in the regime in which the $\mathrm{Ga}(\mathrm{ad})$ atoms are destabilized and the system shows a liquid-like two-dimensional character, a sudden increase of $D$ arises, reaching a value of $0.492 \times 10^{-4} \mathrm{~cm}^{2} / \mathrm{s}$ which amounts to one order of magnitude higher than the value of D $\left(0.382 \times 10^{-5}\right)$ computed at $1100 \mathrm{~K}$.

To get a deeper insight into the destabilization process responsible for the displacement and clustering of $\mathrm{Ga}(\mathrm{ad})$ atoms at the system surface, we made use of the constrained dynamics and the post-processing thermodynamic integration as formalized in the BME method [20]. For our specific problem, we selected as a reaction coordinate $\xi$ the distance between two $\mathrm{Ga}(\mathrm{ad}), \xi=\left|\mathbf{R}_{\mathrm{Ga}(\mathrm{ad})}-\mathbf{R}_{\mathrm{Ga}(\mathrm{ad})}\right|$ and decremented this distance by steps of $0.3 \AA$ from the initial equilibrium value of $6.4 \AA$ up to $\sim 1.9 \AA$ which is the lower bound for a Ga-Ga chemical bond [27]. The average constraint force $f_{\xi}$ becomes the time average of the Lagrange multiplier in front of the constraint which is linearly added to the Car-Parrinello Lagrangean [30]:

$$
f_{\xi}=\left\langle\lambda_{k}\right\rangle_{t}=\frac{\partial F}{\partial \xi}
$$

The free energy profile of the reaction is given by

$$
\Delta F=\int_{\xi_{0}}^{\xi_{f}} f_{\xi} d \xi
$$

The result of this analysis is reported in Fig. 5, where both the free energy (in red) and the total energy (in black) profiles are shown. The latter is computed directly in the usual way as the sum of the DFT total energy plus the ionic kinetic energy (i.e. temperature) of the system.

While controlling the $\mathrm{Ga}(\mathrm{ad})-\mathrm{Ga}(\mathrm{ad})$ relative distance during the BME simulation, $\mathrm{Ga}(\mathrm{ad})$ are free to move along all other directions orthogonal to the constraint. Thus, on the total 
energy space, they can explore other local minima which are not visible from the free energy landscape because not represented by the selected constraint. This is the origin and the reason of the small energy barrier $(\sim 0.53 \mathrm{eV})$ at $5.82 \AA$ and the subsequent local minimum at 5.29 $\AA$ on the black line in Fig. 5, as opposed to a monotonic increase of the free energy profile in this same range.

\subsection{Discussion}

We have reported part of the present results in our previous work [31]. In addition to the reported result, to rationalize the destabilization of the $\mathrm{Ga}(\mathrm{ad})$ atoms at $1300 \mathrm{~K}$, we have extracted from the trajectory the velocities of each atom and plotted the resulting local average temperature as a function of the layer to which atoms belong. The result, summarized by Fig. 6, supports the notion of higher thermal fluctuations of surface atoms we inferred in the former paragraph.

More precisely, since the exposed surface layer and, even more, the $\mathrm{Ga}(\mathrm{ad})$ atoms are less bound than all the rest of the (bulk) system, they uptake most of the thermal energy. Indeed, their local temperatures are on average higher $(1396.9 \mathrm{~K}$ for the $\mathrm{Ga}(\mathrm{ad})$ and $1332.2 \mathrm{~K}$ for the surface GaN layer) than the overall $1300 \mathrm{~K}$, which represents the global temperature of the system. Even more important, instantaneous fluctuations of the temperature during the dynamics affect mainly the $\mathrm{Ga}(\mathrm{ad})$ atoms that can experience sudden increases up to $\sim 1500$ $\mathrm{K}(=0.19 \mathrm{eV})$. The high frequency (see Fig. 1) at which these fluctuations occur affects the $\mathrm{Ga}(\mathrm{ad})$ atoms which are destabilized from the sites where they are initially located. This, in turn, promotes the cleavage of the long bonds of $\mathrm{Ga}(\mathrm{ad})$ with the substrate and favors the formation of the Ga two-dimensional liquid state at the surface of the growing GaN. We remark that the sudden drop in the temperature at the bottom layer in Fig. 6 is just an artifact due to the fixing of these atoms to the bulk crystallographic position as explained in the methodological section. However, the trend of the temperature gradient as a function of the thickness (Fig. 6) justifies the choice of keeping into account six layers in order to have an unbiased thermal motion of at least the top four layers, the last one being our exposed surface. The free energy profile obtained from the BME simulations, accompanied by the total energy profile, gives another useful information. In terms of pure bond energy formation, i.e. our 
selected reaction coordinate, a barrier of about $1.0 \mathrm{eV}$ has to be overcome to bring two $\mathrm{Ga}(\mathrm{ad})$ close to each other. This value accounts for the cleavage of the three $\mathrm{Ga}(\mathrm{ad})-\mathrm{Ga}$ surface chemical bonds of at least one of the two $\mathrm{Ga}(\mathrm{ad})$ included in the reaction coordinate, plus the approach of the two $\mathrm{Ga}(\mathrm{ad})$ on a surface which is full of local minima, more or less shallow, where a Ga(ad) can stabilize temporarily. Yet, the destabilization resulting from the enhanced temperature discussed above greatly contributes to the overcoming of activation barriers and makes easily accessible neighbor stable local minima that eventually Ga(ad) can visit at $1300 \mathrm{~K}$ (or above) giving rise to the diffusion and clustering phenomena. In the BME free (and total) energy profile, at a Ga-Ga distance of $2.17 \AA$ a new minimum is reached, corresponding to a typical Ga dimer [27], providing a full microscopic picture of the aggregation process at the growing $\mathrm{GaN}$ surface.

Based on the results of our simulations, we propose some general comments about the atomic scale mechanism regulating the epitaxial growth. These observations can provide some additional hint and serve as a guideline for further simulations. On a first instance, we remark that the epitaxial growth has been so far believed to occur by destruction of individual chemical bonds on the solid surface and the subsequent formation of new chemical bonds with arriving atomic and molecular species. However, the present theoretical finding of the two-dimensional liquid-like phase leads to a new picture: At the growth temperature, the solid Ga-covered surface transforms to a two-dimensional liquid surface; the $\mathrm{N}$ species then easily intervenes into the subsurface region and bridges the Ga adatom in the liquid phase and the Ga atom in the sub surface layer as sketched in Fig. 7. This is the formation of a new Ga-N network. This picture is consistent with a well-known fact, namely the fact that the temperature range in which the thin films grow is very narrow: The lower limit of this temperature range is the temperature at which the liquid-phase is formed, whereas the upper limit is the temperature at which the liquid evaporates into the gas phase.

\section{Conclusions}

By resorting to first-principle dynamical computational methods, we investigated the microscopic behavior of a Ga-rich GaN surface at different thermodynamic conditions, with special emphasis on the typical experimental growth temperature of $1300 \mathrm{~K}$. An analysis of 
both the surface atomic distribution, in terms of PCFs, and the motion of the atoms at the various increasing temperatures, in terms of diffusion coefficients, has revealed that at the experimental growth temperature a two-dimensional liquid state is realized. When this regime is reached, the Ga adatoms incorporated at the surface of the growing structure are rapidly destabilized and their weak bonds with the surface underneath are easily broken. In these conditions $\mathrm{Ga}$ adatoms can diffuse and migrate on the surface, forming and disrupting continuously Ga clusters, as observed in non-polar liquid systems. Yet, they remain confined on the surface, without evaporating into a gas phase, thus remaining available to a subsequent formation of chemical bond with incoming N. The weakening and disruption of the $\mathrm{Ga}(\mathrm{ad})$ bond with the surface is also expected to facilitate the incorporation of $\mathrm{N}$ for the formation of the $\mathrm{GaN}$ network on the growing system.

\section{Acknowledgments}

This work was partly supported by the MEXT, Japan under the research projects "Social and Scientific Priority Issue to be Tackled by Using Post-K Computer" and "Program for Research and Development of Next-Generation Semiconductors to Realize an EnergySaving Society", and also under the contract number $18 \mathrm{H} 03873$ of the grants-in-aid. M.B. thanks Pôle HPC Equip@Meso-CPER at the University of Strasbourg, and Grand Equipement National de Calcul Intensif(GENCI) under allocation DARI-A6 A0060906092. Computations were performed partly at Supercomputer Centers of ISSP, University of Tokyo and of IMS, National Institutes of Natural Sciences, and at K-computer at RIKEN. 


\section{References}

[1] B. Rackauskas, M. J. Uren, T. Kachi, and M. Kuball, Microelectron. Reliab. 95, 48 (2019).

[2] I. Akasaki, Rev. Mod. Phys. 87, 1119 (2015).

[3] S. Nakamura, Rev. Mod. Phys. 87, 1139 (2015).

[4] H. Amano, Rev. Mod. Phys. 87, 1133 (2015).

[5] K. Nagamatsu, S. Nitta, Z. Ye, H. Nagao, S. Miki, Y. Honda, and H. Amano, Phys. Status Solidi 254, 1600737 (2017).

[6] Y. Kangawa, T. Akiyama, T. Ito, K. Shiraishi, and T. Nakayama, Materials (Basel). 6, 3309 (2013).

[7] A. Kusaba, Y. Kangawa, P. Kempisty, H. Valencia, K. Shiraishi, Y. Kumagai, K. Kakimoto, and A. Koukitu, Jpn. J. Appl. Phys. 56, (2017).

[8] K. M. Bui, J.-I. Iwata, Y. Kangawa, K. Shiraishi, Y. Shigeta, and A. Oshiyama, J. Phys. Chem. C 122, 24665 (2018).

[9] K. M. Bui, J.-I. Iwata, Y. Kangawa, K. Shiraishi, Y. Shigeta, and A. Oshiyama, J. Cryst. Growth 507, 421 (2019).

[10] P. Hohenberg and W. Kohn, Phys. Rev. 136, B864 (1964).

[11] W. Kohn and L. J. Sham, Phys. Rev. 140, A1133 (1965).

[12] R. Car and M. Parrinello, Phys. Rev. Lett. 55, 2471 (1985).

[13] M. Boero and A. Oshiyama, in Encycl. Nanotechnol. (Springer Netherlands, Dordrecht, 2015), pp. 1-10.

[14] D. Marx and J. Hutter, Ab Initio Molecular Dynamics (Cambridge University Press, Cambridge, 2009).

[15] C. M. für F. S. 1997-2001. 1) CPMD, http://www.cpmd.org/, Copyright IBM Corp 1990-2019, (n.d.).

[16] J.-I. Iwata, D. Takahashi, A. Oshiyama, T. Boku, K. Shiraishi, S. Okada, and K. Yabana, J. Comput. Phys. 229, 2339 (2010). 
[17] Y. Hasegawa, J.-I. Iwata, M. Tsuji, D. Takahashi, A. Oshiyama, K. Minami, T. Boku, H. Inoue, Y. Kitazawa, I. Miyoshi, and M. Yokokawa, Int. J. High Perform. Comput. Appl. 28, 335 (2014).

[18] K. Uchida, S. Furuya, J.-I. Iwata, and A. Oshiyama, Phys. Rev. B 90, 155451 (2014).

[19] G. Bussi, D. Donadio, and M. Parrinello, J. Chem. Phys. 126, 014101 (2007).

[20] M. Sprik and G. Ciccotti, J. Chem. Phys. 109, 7737 (1998).

[21] J. P. Perdew, K. Burke, and M. Ernzerhof, Phys. Rev. Lett. 77, 3865 (1996).

[22] S. Kümmel and L. Kronik, Rev. Mod. Phys. 80, 3 (2008).

[23] S. Grimme, J. Comput. Chem. 27, 1787 (2006).

[24] S. Goedecker, M. Teter, and J. Hutter, Phys. Rev. B 54, 1703 (1996).

[25] M. S. Green and M. S., J. Chem. Phys. 22, 398 (1954).

[26] R. Kubo, J. Phys. Soc. Japan 12, 570 (1957).

[27] Y. Xie, R. S. Grev, J. Gu, H. F. Schaefer, P. v. R. Schleyer, J. Su, X. W. Li, and G. H. Robinson, J. Am. Chem. Soc. 120, 3773 (1998).

[28] J. G. Kirkwood and E. M. Boggs, J. Chem. Phys. 10, 394 (1942).

[29] Y. Zhao, Z. Wu, and W. Liu, Phys. A Stat. Mech. Its Appl. 390, 2812 (2011).

[30] M. Boero, A. Bouzid, S. Le Roux, B. Ozdamar, and C. Massobrio, in (Springer, Cham, 2015), pp. 33-55.

[31] K. M. Bui, M. Boero, K. Shiraishi, and A. Oshiyama, Extended Abstracts of the 2019 International Conference on Solid State Devices and Materials, Nagoya (2019), pp281-282. 


\section{Figure Captions}

Fig. 1. (color online) Temperature increase during the heating phase. The inset shows the surface of the GaN system simulated. $\mathrm{Ga}$ and $\mathrm{N}$ atoms of the substrate are colored in pink and blue, respectively, whereas the Ga adatom $[\mathrm{Ga}(\mathrm{ad})]$ exposed at the surface are evidenced in yellow.

Fig. 2. (color online) Evolution of the $\mathrm{Ga}(\mathrm{ad})-\mathrm{Ga}(\mathrm{ad})$ pair correlation function (equation (1)) during the heating process. The initial distance of $6.4 \AA$ corresponds to the relaxed system from which dynamical simulations were started.

Fig. 3. (color online) Pair correlation function (PCF) for the Ga(ad)-Ga(ad) distribution at $1300 \mathrm{~K}$ (lower panel) and corresponding coordination number as obtained upon integration of this PCF. The insets show the structures corresponding to a dimer or a trimer of Ga atoms diffusing on the GaN (0001) surface. The color code for atoms is identical to Fig. 1.

Fig. 4. (color online) Diffusion coefficient of a Ga adatom on Ga-rich GaN (0001) surface as a function of the inverse temperature $1 / T$ for the temperature range (300-1300 K) investigated in the present work. The dashed red line shows the Arrhenius plot obtained by excluding the point at $1300 \mathrm{~K}$, where the system deviates from the standard trend.

Fig. 5. (color online) Free (red line with diamonds) and total (black line with dots) energy profiles for the destabilization process of $\mathrm{Ga}$ adatom located at the initial position (right end), resulting in the formation of a Ga-Ga dimer on the Ga-rich (0001) GaN surface (left end). The two stick-and-balls snapshots show the initial step (right panel) and the final Ga-Ga dimer (left panel) with the same color code for atoms of Fig. 1. The green trajectories sketch the path followed by the diffusing $\mathrm{Ga}(\mathrm{ad})$ during the simulation. 
Fig. 6. (color online) Temperature profile (right panel) as a function of the depth in the slab (left panel). The upper point at 0 refers to the $\mathrm{Ga}(\mathrm{ad})$ adatoms, the second, labeled as 1 , to the surface GaN first layer and the following numbers to the descending layers underneath. The color code adopted is identical to all former figures for $\mathrm{Ga}(\mathrm{ad}), \mathrm{Ga}$ and $\mathrm{N}$. White spheres indicate, instead, the saturating $\mathrm{H}$ atoms at the bottom layer fixed to the bulk crystallographic positions.

Fig. 7. (color online) Schematic of $\mathrm{N}$ incorporation under two-dimensional Ga liquid on the $\mathrm{GaN}$ films. The color code adopted is identical to all former figures 


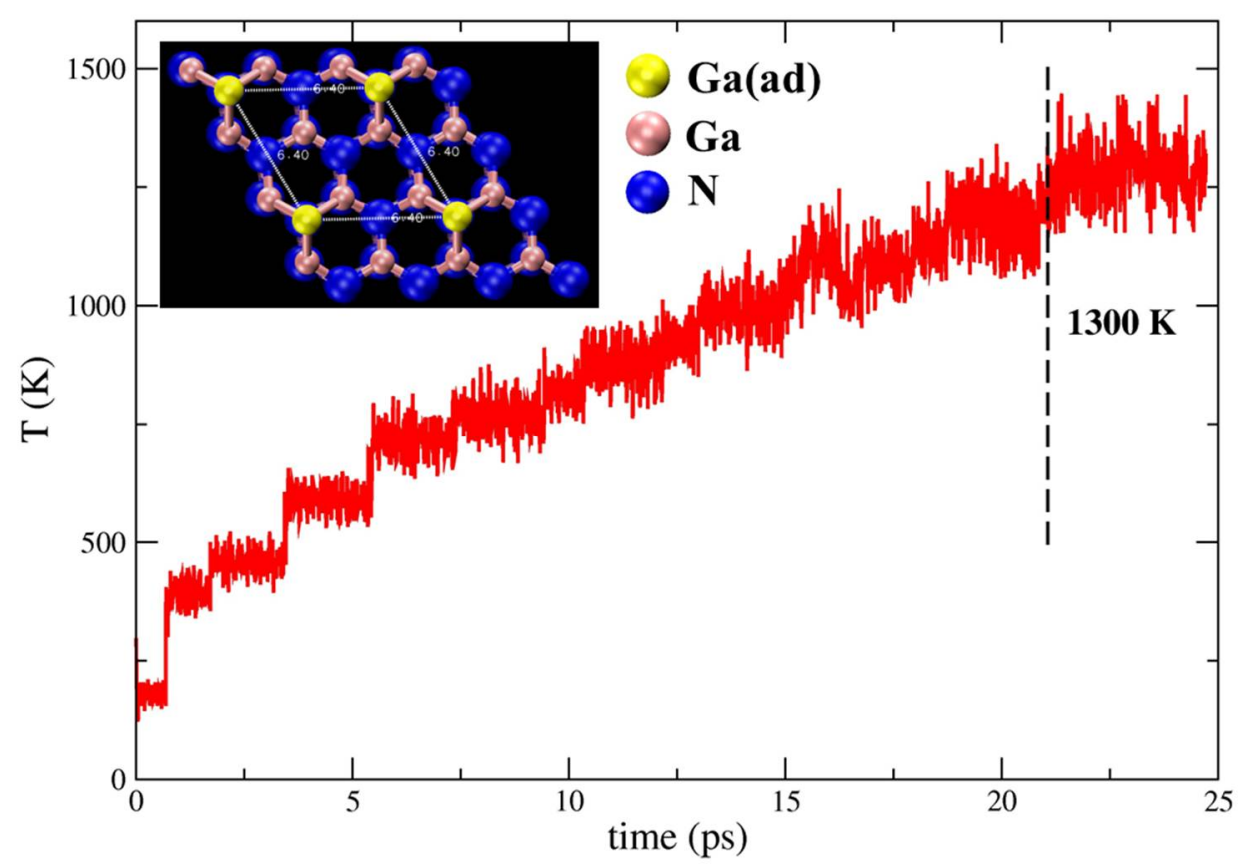

Fig. 1.

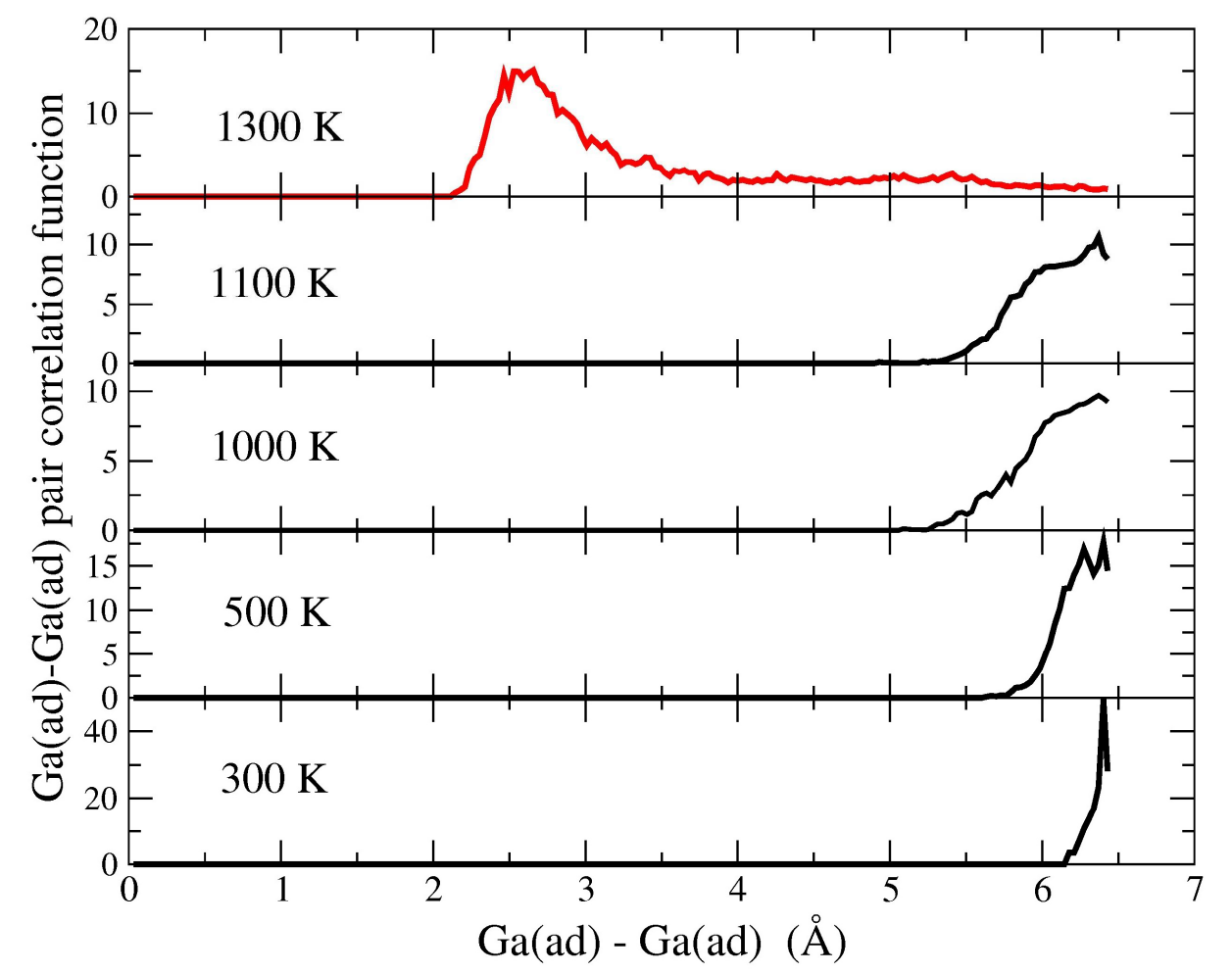

Fig. 2. 


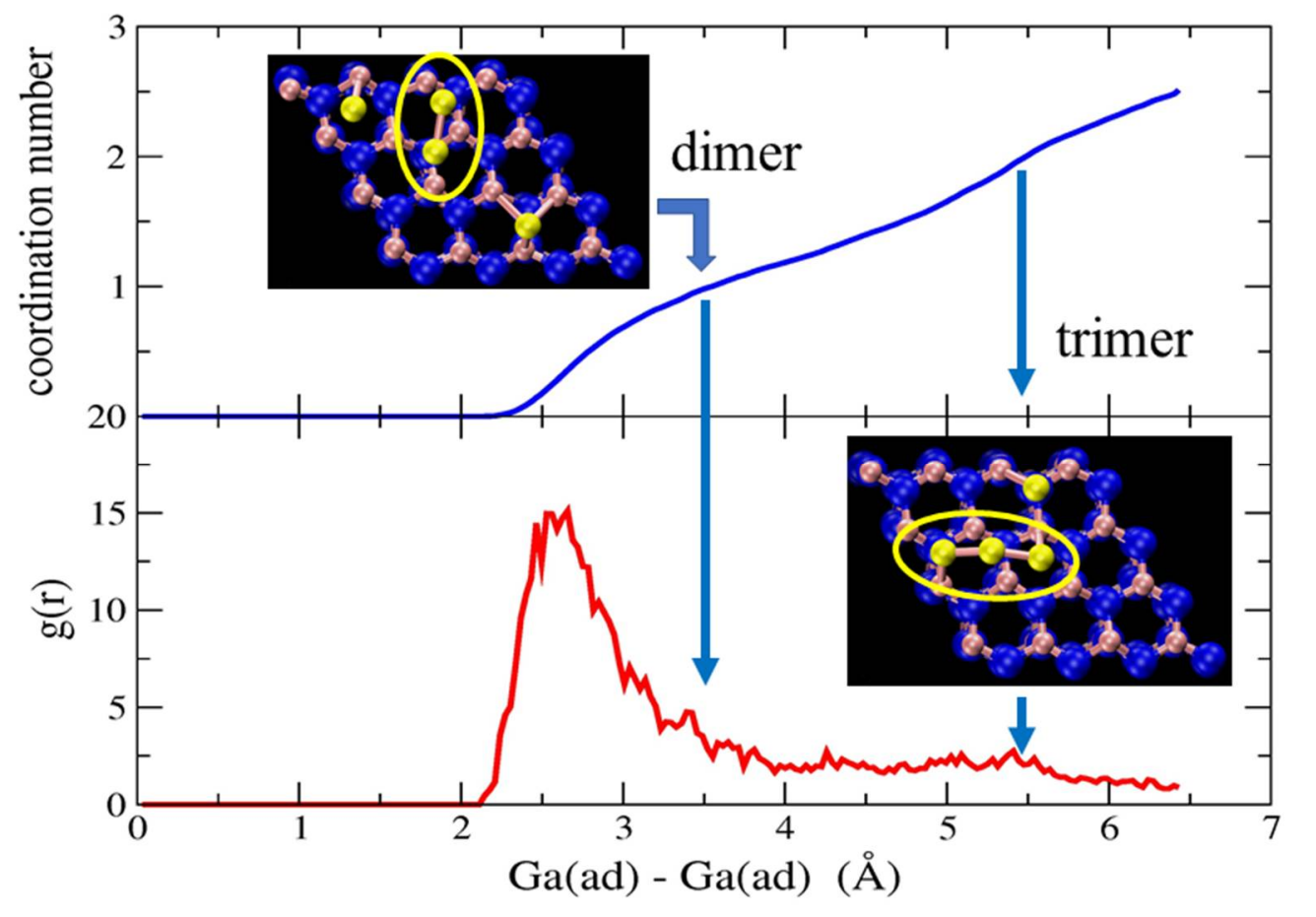

Fig.3.

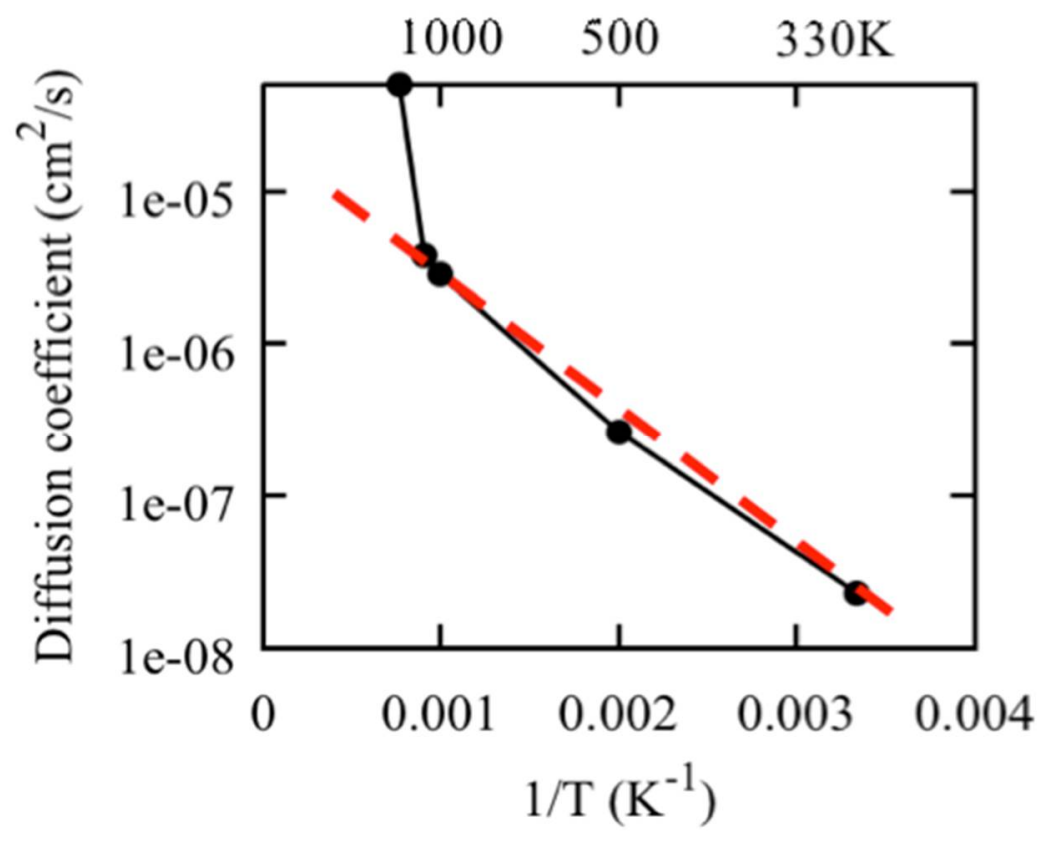

Fig. 4. 


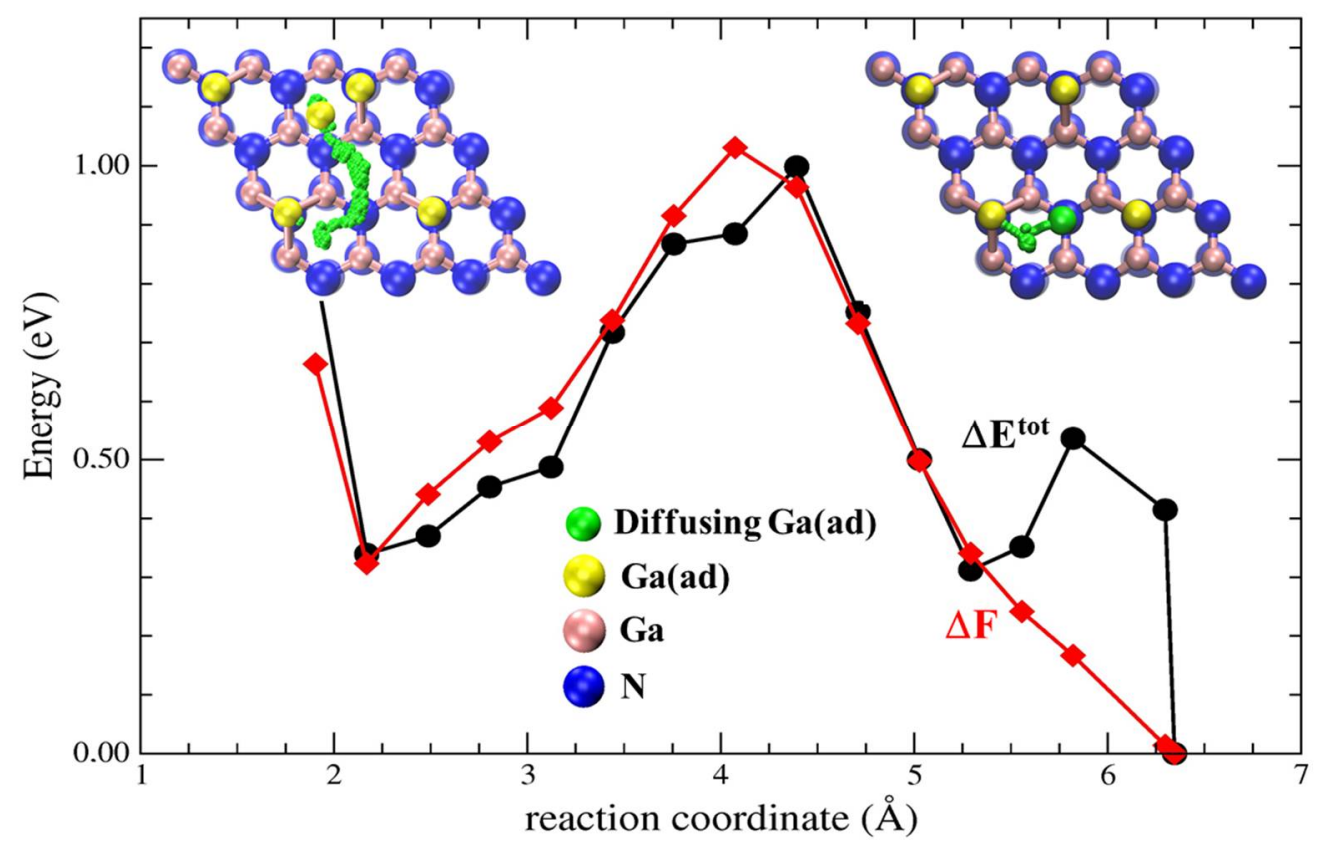

Fig. 5.

$\mathrm{T}(\mathrm{K})$

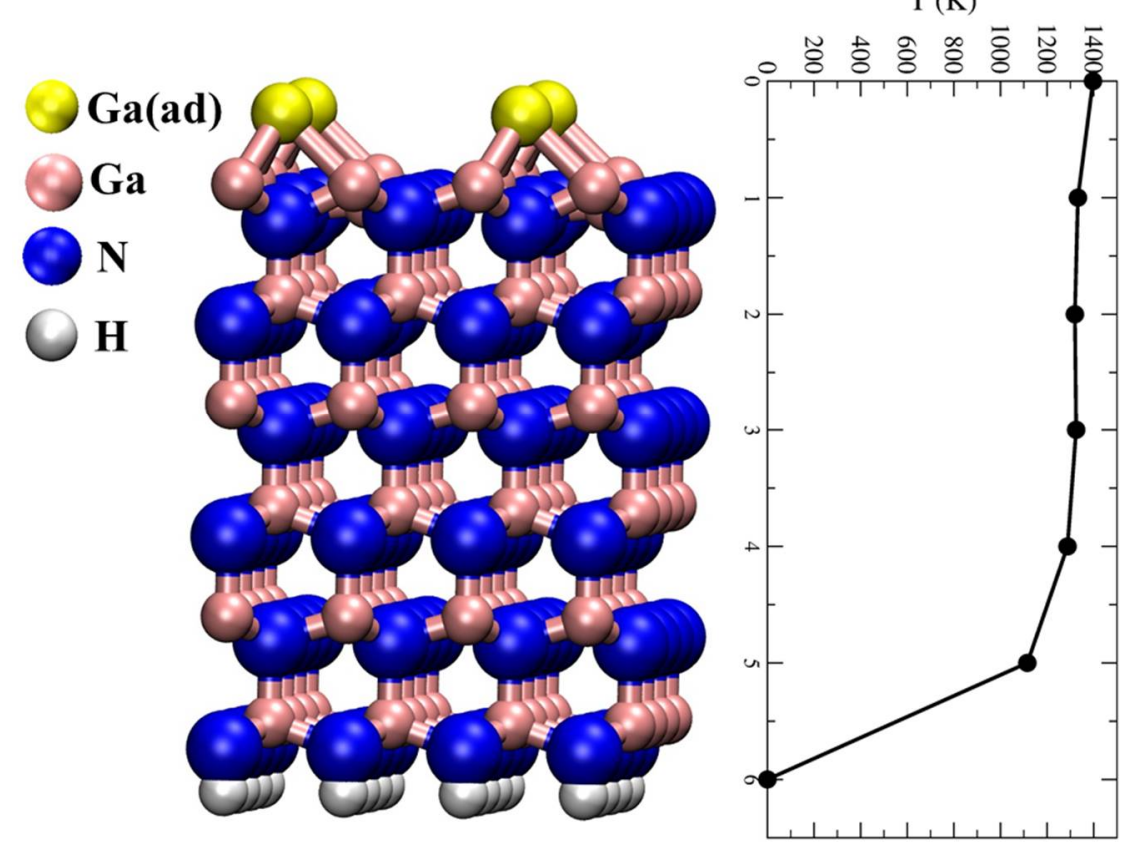

Fig. 6. 


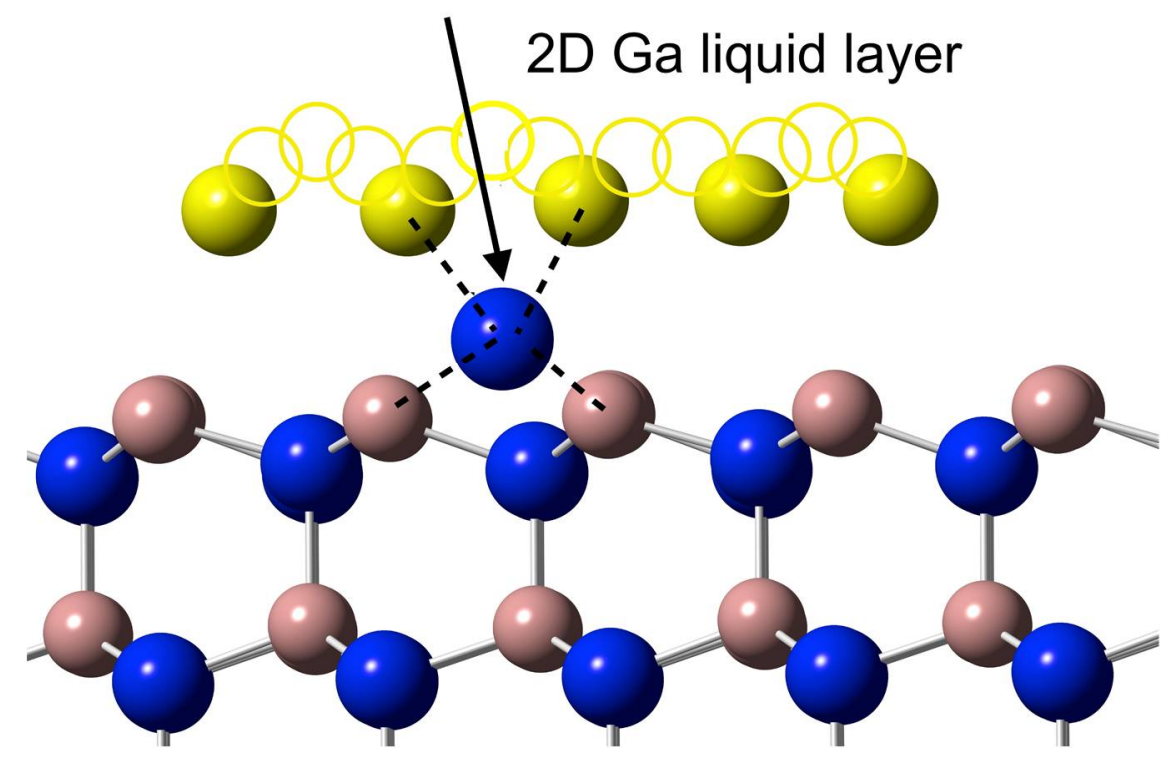

Fig. 7. 